\title{
THE ROLE OF THE AHLFORS MAPPING IN THE THEORY OF KERNEL FUNCTIONS IN THE PLANE
}

\author{
SteVen R. BeLL*
}

\begin{abstract}
We describe recent results that establish a close relationship between the Ahlfors mapping function associated to an $n$-connected domain in the plane and the Bergman and Szegö kernels of the domain. The results show that the Ahlfors mapping plays a role in the multiply connected setting very similar to that of the Riemann mapping in the simply connected case. We also describe how the Ahlfors map is connected to the Poisson and other kernels.
\end{abstract}

\section{INTRODUCTION}

The Bergman and Szegö kernel functions associated to a simply connected domain $\Omega$ in the complex plane are easily expressed in terms of a single Riemann mapping function associated to the domain. Indeed, if $a$ is a point in $\Omega$ and $f_{a}(z)$ is the Riemann mapping function mapping $\Omega$ one-to-one onto the unit disc $D_{1}(0)$ with $f_{a}(a)=0$ and $f_{a}^{\prime}(a)>0$, then the Szegö kernel $S(z, w)$ is given by

$$
S(z, w)=\frac{c S(z, a) \overline{S(w, a)}}{1-f_{a}(z) \overline{f_{a}(w)}},
$$

and the Bergman kernel $K(z, w)$ is given by

$$
K(z, w)=\frac{4 \pi c^{2} S(z, a)^{2} \overline{S(w, a)^{2}}}{\left(1-f_{a}(z) \overline{f_{a}(w)}\right)^{2}},
$$

where $c=1 / S(a, a)$ in both the formulas. The Szegö kernel could be eliminated from the right hand side of these formulas by noting that $f_{a}^{\prime}(z)=2 \pi c S(z, a)^{2}$. However, we shall see that the formulas above have natural generalizations to multiply connected domains.

These identities reveal that, not only can Riemann maps be computed by means of kernel functions, but kernel functions can be computed by means of Riemann maps. In this paper, I shall describe analogous results for $n$-connected domains that show that the Bergman and Szegö kernels are simple rational combinations of an Ahlfors map and $n$ other basic functions of one variable related to the zeroes of the Ahlfors map.

There are many ways in which the Ahlfors map can be thought of as the "Riemann mapping function for multiply connected domains." Indeed, the Ahlfors map 
associated to a point $a$ in a multiply connected domain $\Omega$ is the unique holomorphic function $f$ mapping $\Omega$ into the unit disc that makes $f^{\prime}(a)>0$ and as large as possible. Furthermore, the Ahlfors map also has mapping properties analogous to the Riemann map; it maps $\Omega$ onto the unit disc and maps each boundary curve of $\Omega$ one-to-one onto the unit circle (see $\S 2$ for more details). We shall see that, from the point of view of kernel functions, the Ahlfors map also takes on the role of a "Riemann map in multiply connected domains."

In the last section of this paper, I describe the relationship between the Ahlfors map and the Poisson kernel, the Garabedian kernel, and the complimentary kernel to the Bergman kernel.

2. The Ahlfors map and zeroes of the Szegö kernel. We shall study the kernel functions on a finitely connected domain in the plane such that no boundary component reduces to a point. Such a domain can be mapped biholomorphically to a bounded domain $\Omega$ with $C^{\infty}$ smooth boundary, i.e., a bounded domain whose boundary $b \Omega$ is given by finitely many non-intersecting $C^{\infty}$ simple closed curves.

In order to continue, we must list some basic facts about the kernel functions. Suppose that $\Omega$ is a bounded $n$-connected domain in the plane with $C^{\infty}$ smooth boundary. Let $\gamma_{j}, j=1, \ldots, n$, denote the $n$ non-intersecting $C^{\infty}$ simple closed curves which define the boundary of $\Omega$, and suppose that $\gamma_{j}$ is parameterized in the standard sense by $z_{j}(t), 0 \leq t \leq 1$. Let $T(z)$ be the $C^{\infty}$ function defined on $b \Omega$ such that $T(z)$ is the complex number representing the unit tangent vector at $z \in b \Omega$ pointing in the direction of the standard orientation. This complex unit tangent vector function is characterized by the equation $T\left(z_{j}(t)\right)=z_{j}^{\prime}(t) /\left|z_{j}^{\prime}(t)\right|$.

We shall let $A^{\infty}(\Omega)$ denote the space of holomorphic functions on $\Omega$ that are in $C^{\infty}(\bar{\Omega})$. The space of complex valued functions on $b \Omega$ that are square integrable with respect to arc length measure $d s$ will be denoted by $L^{2}(b \Omega)$. We shall let $H^{2}(b \Omega)$ denote the space of functions in $L^{2}(b \Omega)$ that represent the $L^{2}$ boundary values of holomorphic functions on $\Omega$ (as described in [1]) and we shall call $H^{2}(b \Omega$ ) the Hardy space. The inner product associated to $L^{2}(b \Omega)$ shall be written

$$
\langle u, v\rangle_{b \Omega}=\int_{b \Omega} u \bar{v} d s .
$$

For each fixed point $a \in \Omega$, the Szegö kernel $S(z, a)$, as a function of $z$, extends to the boundary to be a function in $A^{\infty}(\Omega)$. Furthermore, $S(z, a)$ has exactly $(n-1)$ zeroes as a function of $z$ in $\Omega$ (counting multiplicities) and does not vanish at any points $z$ in the boundary of $\Omega$. Furthermore, $S(z, w)$ is in $C^{\infty}((\bar{\Omega} \times \bar{\Omega})-\{(z, z)$ : $z \in b \Omega\})$ as a function of $(z, w)$.

The Garabedian kernel $L(z, a)$ is a kernel related to the Szegö kernel via the identity

$$
\frac{1}{i} L(z, a) T(z)=S(a, z) \quad \text { for } z \in b \Omega \text { and } a \in \Omega
$$

For fixed $a \in \Omega$, the kernel $L(z, a)$ is a holomorphic function of $z$ on $\Omega-\{a\}$ with a simple pole at $a$ with residue $1 /(2 \pi)$. Furthermore, as a function of $z, L(z, a)$ extends to the boundary and is in the space $C^{\infty}(\bar{\Omega}-\{a\})$. In fact, $L(z, a)$ extends to be in $C^{\infty}((\bar{\Omega} \times \bar{\Omega})-\{(z, z): z \in \bar{\Omega}\})$. Also, $L(z, a)$ is non-zero for all $(z, a)$ in $\bar{\Omega} \times \Omega$ with $z \neq a$. 
The kernel $S(z, w)$ is holomorphic in $z$ and antiholomorphic in $w$ on $\Omega \times \Omega$, and $L(z, w)$ is holomorphic in both variables for $z, w \in \Omega, z \neq w$. We note here that $S(z, z)$ is real and positive for each $z \in \Omega$, and that $S(z, w)=\overline{S(w, z)}$ and $L(z, w)=-L(w, z)$. Also, the Szegö kernel reproduces holomorphic functions in the sense that

$$
h(a)=\langle h, S(\cdot, a)\rangle_{b \Omega}
$$

for all $h \in H^{2}(b \Omega)$ and $a \in \Omega$.

Given a point $a \in \Omega$, the Ahlfors map $f_{a}$ associated to the pair $(\Omega, a)$ is a proper holomorphic mapping of $\Omega$ onto the unit disc. It is an $n$-to-one mapping (counting multiplicities), it extends to be in $A^{\infty}(\Omega)$, and it maps each boundary curve $\gamma_{j}$ oneto-one onto the unit circle. Furthermore, $f_{a}(a)=0$, and $f_{a}$ is the unique function mapping $\Omega$ into the unit disc maximizing the quantity $\left|f_{a}^{\prime}(a)\right|$ with $f_{a}^{\prime}(a)>0$. The Ahlfors map is related to the Szegö kernel and Garabedian kernel via

$$
f_{a}(z)=\frac{S(z, a)}{L(z, a)} .
$$

Note that $f_{a}^{\prime}(a)=2 \pi S(a, a) \neq 0$. Because $f_{a}$ is $n$-to-one, $f_{a}$ has $n$ zeroes. The simple pole of $L(z, a)$ at $a$ accounts for the simple zero of $f_{a}$ at $a$. The other $n-1$ zeroes of $f_{a}$ are given by $(n-1)$ zeroes of $S(z, a)$ in $\Omega$. Let $a_{1}, a_{2}, \ldots, a_{n-1}$ denote these $n-1$ zeroes (counted with multiplicity). I proved in [2] (see also [1, page 105]) that, if $a$ is close to one of the boundary curves, the zeroes $a_{1}, \ldots, a_{n-1}$ become distinct simple zeroes. It follows from this result that, for all but at most finitely many points $a \in \Omega, S(z, a)$ has $n-1$ distinct simple zeroes in $\Omega$ as a function of $z$.

3. Nearly orthogonal power series on multiply connected domains. The Ahlfors function gives rise to a particularly nice basis for the Hardy space of an $n$-connected domain with $C^{\infty}$ smooth boundary. We shall use the notation that we set up in the preceding section. We assume that $a \in \Omega$ is a fixed point in $\Omega$ that has been chosen so that the $n-1$ zeroes, $a_{1}, \ldots, a_{n-1}$, of $S(z, a)$ are distinct and simple. We shall let $a_{0}$ denote $a$ and we shall use the shorthand notation $f(z)$ for the Ahlfors map $f_{a}(z)$.

It was proved in [2] that the set of functions

$$
h_{i k}(z)=S\left(z, a_{i}\right) f(z)^{k},
$$

where $0 \leq i \leq n-1$ and $k \geq 0$, forms a basis for the Hardy space $H^{2}(b \Omega)$ and that

$$
\left\langle h_{i k}, h_{j m}\right\rangle_{b \Omega}= \begin{cases}0, & \text { if } k \neq m, \\ S\left(a_{j}, a_{i}\right), & \text { if } k=m .\end{cases}
$$

I shall prove this result here in order to demonstrate an interesting connection between the Ahlfors map and "power series" on multiply connected domains. First, I will show that the set of functions above spans a dense subset of $H^{2}(b \Omega)$. Indeed, suppose that $g \in H^{2}(b \Omega)$ is orthogonal to the span. Notice that the reproducing property of the Szego" kernel yields that

$$
\left\langle g, S\left(\cdot, a_{j}\right)\right\rangle_{b \Omega}=g\left(a_{j}\right),
$$

and therefore $g$ vanishes at $a_{0}, a_{1}, \ldots, a_{n-1}$. Suppose we have shown that $g$ vanishes to order $m$ at each $a_{j}, j=0,1, \ldots, n-1$. It follows that $g / f^{m}$ has removable 
singularities at each $a_{j}$ and so it can be viewed as an element of $H^{2}(b \Omega)$. The value of $g / f^{m}$ at $a_{j}$ is $\frac{1}{m !} g^{(m)}\left(a_{j}\right) / f^{\prime}\left(a_{j}\right)^{m}$. Since $|f(z)|=1$ when $z \in b \Omega$, it follows that $1 / f(z)=\overline{f(z)}$ when $z \in b \Omega$, and we may write

$$
\left\langle g, S\left(\cdot, a_{j}\right) f^{m}\right\rangle_{b \Omega}=\left\langle g / f^{m}, S\left(\cdot, a_{j}\right)\right\rangle_{b \Omega}=\frac{1}{m !} g^{(m)}\left(a_{j}\right) / f^{\prime}\left(a_{j}\right)^{m} .
$$

(The last equality follows from the reproducing property of the Szegö kernel.) We conclude that $g$ vanishes to order $m+1$ at each $a_{j}$. By induction, $g$ vanishes to infinite order at each $a_{j}$ and hence, $g \equiv 0$. This proves the density.

To prove (3.1), let us suppose first that $k>m$. The fact that $\bar{f}=1 / f$ on $b \Omega$ and the reproducing property of the Szegö kernel now yield that

$$
\begin{gathered}
\left\langle h_{i k}, h_{j m}\right\rangle_{b \Omega}=\int_{z \in b \Omega} S\left(z, a_{i}\right) f(z)^{k-m} \overline{S\left(z, a_{j}\right)} d s= \\
\int_{z \in b \Omega} S\left(a_{j}, z\right)\left[S\left(z, a_{i}\right) f(z)^{k-m}\right] d s=S\left(a_{j}, a_{i}\right) f\left(a_{j}\right)^{k-m} .
\end{gathered}
$$

The identity now follows because $f\left(a_{j}\right)=0$ for all $j$. If $k=m$, then

$$
\left\langle h_{i k}, h_{j m}\right\rangle_{b \Omega}=\int_{z \in b \Omega} S\left(a_{j}, z\right) S\left(z, a_{i}\right) d s=S\left(a_{j}, a_{i}\right),
$$

and identity (3.1) is proved. It is now easy to see that the functions $h_{i k}$ are linearly independent. Indeed, identity (3.1) reveals that we need only check that, for fixed $k$, the $n$ functions $h_{i k}, i=0,1, \ldots, n-1$, are linearly independent, and this is true because a relation of the form

$$
\sum_{i=0}^{n-1} C_{i} S\left(z, a_{i}\right) \equiv 0
$$

implies, via the reproducing property of the Szegö kernel, that every function $g$ in the Hardy space satisfies

$$
\sum_{i=0}^{n-1} \overline{C_{i}} g\left(a_{i}\right)=0
$$

and it is easy to construct polynomials $g$ that violate such a condition.

To obtain a formula for the Szegö kernel on $\Omega$, we next orthonormalize the sequence $\left\{h_{i k}\right\}$ via the Gram-Schmidt procedure. Identity (3.1) shows that the functions in the sequence are orthogonal for different values of $k$, and so our task is merely to orthonormalize the $n$ functions $h_{i k}, i=0,1, \ldots, n-1$ for each $k$. We obtain an orthonormal set $\left\{H_{i k}\right\}$ given by

$$
\begin{gathered}
H_{0 k}(z)=b_{00} S(z, a) f(z)^{k} \quad \text { and } \\
H_{i k}(z)=\sum_{j=1}^{i} b_{i j} S\left(z, a_{j}\right) f(z)^{k}, \quad i=1, \ldots, n-1,
\end{gathered}
$$


where $b_{i i} \neq 0$ for each $i=0,1, \ldots, n-1$. Because $|f|=1$ on $b \Omega$, it follows that the coefficients $b_{i j}$ do not depend on $k$. Notice that $H_{i k}$ does not contain a term involving $S(z, a)$ if $i>0$ because of (3.1) and the fact that $S\left(a_{i}, a\right)=0$.

The Szegö kernel can be written in terms of our orthonormal basis as

$$
S(z, w)=\sum_{i=0}^{n-1} \sum_{k=0}^{\infty} H_{i k}(z) \overline{H_{i k}(w)} .
$$

The geometric sum

$$
\sum_{k=0}^{\infty} f(z)^{k} \overline{f(w)^{k}}=\frac{1}{1-f(z) \overline{f(w)}}
$$

can be factored from the expression for $S(z, w)$ to yield the formula,

$$
S(z, w)=\frac{1}{1-f(z) \overline{f(w)}}\left(c_{0} S(z, a) \overline{S(w, a)}+\sum_{i, j=1}^{n-1} c_{i j} S\left(z, a_{i}\right) \overline{S\left(w, a_{j}\right)}\right) .
$$

We shall now determine the coefficients in this formula. At the moment, we only know that these coefficients exist and that they are given as combinations of the Gram-Schmidt coefficients found above. That $c_{0}=1 / S(a, a)$ can be seen by setting $z=a$ and $w=a$ in (3.2). To determine the coefficients $c_{i j}$, suppose $1 \leq k \leq n-1$ and set $w=a_{k}$ in (3.2). Note that $f\left(a_{k}\right)=0$ and that $S\left(a, a_{k}\right)=0$. Hence,

$$
S\left(z, a_{k}\right)=\sum_{i=1}^{n-1}\left(\sum_{j=1}^{n-1} c_{i j} S\left(a_{j}, a_{k}\right)\right) S\left(z, a_{i}\right) .
$$

Such a relation can only be true if

$$
\sum_{j=1}^{n-1} c_{i j} S\left(a_{j}, a_{k}\right)= \begin{cases}1, & \text { if } i=k \\ 0, & \text { if } i \neq k\end{cases}
$$

This shows that the $(n-1) \times(n-1)$ matrix $\left[S\left(a_{j}, a_{k}\right)\right]$ is invertible and that $\left[c_{i j}\right]$ is its inverse. Let us summarize these results in the following theorem.

Theorem 3.1. The Szegö kernel of an n-connected domain is related to the Ahlfors map $f_{a}(z)$ associated to a point $a$ in the domain via the formula

$$
S(z, w)=\frac{1}{1-f_{a}(z) \overline{f_{a}(w)}}\left(c_{0} S(z, a) \overline{S(w, a)}+\sum_{i, j=1}^{n-1} c_{i j} S\left(z, a_{i}\right) \overline{S\left(w, a_{j}\right)}\right)
$$

where $c_{0}=1 / S(a, a)$ and the coefficients $c_{i j}$ are given as the coefficients of the inverse matrix to the matrix $\left[S\left(a_{j}, a_{k}\right)\right]$.

Theorem 3.1 generalizes in a routine manner to any finitely connected domain $\Omega_{1}$ such that no boundary component is a point. Such a domain can be mapped to a finitely connected domain with smooth boundary $\Omega_{2}$ via a biholomorphic mapping 
$\Phi$. The function $\Phi^{\prime}$ has a single valued holomorphic square root on $\Omega_{1}$ (see [1, page 43]) and if we define the Szegö kernel on $\Omega_{1}$ via the natural transformation formula

$$
S_{1}(z, w)=\sqrt{\Phi^{\prime}(z)} S_{2}(\Phi(z), \Phi(w)) \overline{\sqrt{\Phi^{\prime}(w)}},
$$

then it is easy to see that the terms in (3.2) transform in exactly the correct manner in which to make (3.2) valid on $\Omega_{1}$.

We mention that the nearly orthogonal basis $h_{i k}(z)=S\left(z, a_{i}\right) f(z)^{k}$ defined above can be used to expand a holomorphic function on $\Omega$ in a special power series expansion. Indeed, given a homorphic function $G(z)$ in $H^{2}(b \Omega)$, we may write

$$
G(z)=\sum_{i=0}^{n-1} \sum_{k=0}^{\infty} b_{i k} S\left(z, a_{i}\right) f(z)^{k}=\sum_{i=0}^{n-1} S\left(z, a_{i}\right) \sum_{k=0}^{\infty} b_{i k} f(z)^{k} .
$$

The coefficients $b_{i j}$ may be computed by means of the inner product on $b \Omega$, or they may also be computed by inductively equating coefficients of Taylor expansions at each of the points $a_{i}, i=0, \ldots, n-1$. The expansion for $G$ can also be written in the form

$$
G(z)=\sum_{i=0}^{n-1} S\left(z, a_{i}\right) H_{i}(f(z))
$$

where the $H_{i}$ are holomorphic on the unit disc. The $n$ linear operators given by the mappings that take $G$ to $H_{i}$ have yet to be studied.

4. Complexity of the kernel functions. Formula (3.2) reveals that the Szegö kernel associated to an $n$-connected domain is composed of the $n+1$ functions, $S(z, a), S\left(z, a_{1}\right), S\left(z, a_{2}\right), \ldots, S\left(z, a_{n-1}\right)$, and $f_{a}(z)$. (Note that because $f_{a}(z)=$ $S(z, a) / L(z, a)$, we may replace $f_{a}(z)$ in this list of functions by $L(z, a)$ if we like.) We shall now see that the Bergman kernel of an $n$-connected domain in the plane is composed of the same basic functions that comprise the Szegö kernel.

The Bergman kernel $K(z, w)$ is related to the Szegö kernel via the identity

$$
K(z, w)=4 \pi S(z, w)^{2}+\sum_{i, j=1}^{n-1} A_{i j} F_{i}^{\prime}(z) \overline{F_{j}^{\prime}(w)}
$$

where the functions $F_{i}^{\prime}(z)$ are classical functions of potential theory described as follows. The harmonic function $\omega_{j}$ which solves the Dirichlet problem on $\Omega$ with boundary data equal to one on the boundary curve $\gamma_{j}$ and zero on $\gamma_{k}$ if $k \neq j$ has a multivalued harmonic conjugate. The function $F_{j}^{\prime}(z)$ is a globally defined single valued holomorphic function on $\Omega$ which is locally defined as the derivative of $\omega_{j}+i v$ where $v$ is a local harmonic conjugate for $\omega_{j}$. The Cauchy-Riemann equations reveal that $F_{j}^{\prime}(z)=2\left(\partial \omega_{j} / \partial z\right)$.

Let $\mathcal{F}^{\prime}$ denote the vector space of functions given by the complex linear span of the set of functions $\left\{F_{j}^{\prime}(z): j=1, \ldots, n-1\right\}$. It is a classical fact that $\mathcal{F}^{\prime}$ is $n-1$ dimensional. Notice that $S\left(z, a_{i}\right) L(z, a)$ is in $A^{\infty}(\Omega)$ because the pole of $L(z, a)$ at $z=a$ is cancelled by the zero of $S\left(z, a_{i}\right)$ at $z=a$. A theorem due to Schiffer (see $[5,1,2])$ states that the $n-1$ functions $S\left(z, a_{i}\right) L(z, a), i=1, \ldots, n-1$ form a basis for $\mathcal{F}^{\prime}$. We may now write

$$
K(z, w)=4 \pi S(z, w)^{2}+\sum_{i, j=1}^{n-1} \lambda_{i j} S\left(z, a_{i}\right) L(z, a) \overline{S\left(w, a_{j}\right) L(w, a)}
$$


which, together with (3.2) allows us to see that the Bergman kernel is composed of exactly the same basic functions that make up the Szegö kernel.

A recipe is given for explicitly computing all the elements appearing in formula (4.1) in [4]. It is interesting that all the elements of the kernel function can be computed by means of one dimensional line integrals and simple linear algebra.

We have proved formula (4.1) on a domain with smooth boundary. If a finitely connected domain $\Omega_{1}$ does not have smooth boundary, and if none of its boundary components are points, there is a conformal mapping $\Phi$ of $\Omega_{1}$ onto a domain $\Omega_{2}$ whose boundary is smooth. The transformation formula for the Bergman kernels under biholomorphic mappings,

$$
K_{1}(z, w)=\Phi^{\prime}(z) K_{2}(\Phi(z), \Phi(w)) \overline{\Phi^{\prime}(w)},
$$

together with the transformation formula for the Szegö kernels (3.3), can then be used to show that (4.1) is valid on $\Omega_{1}$.

5. The Ahlfors map and other kernel functions. I showed in [3] (see also [1]) how the Szegó projection can be used to solve the Dirichlet problem. The method gives rise to a formula for the Poisson kernel of a bounded $n$-connected domain $\Omega$ with $C^{\infty}$ smooth boundary in terms of the Szegö kernel (see [2]). The Poisson kernel $p(z, w)$ is given by

$$
p(a, w)=\frac{|S(w, a)|^{2}}{S(a, a)}+\sum_{j=1}^{n-1}\left(\omega_{j}(a)-\lambda_{j}(a)\right) \mu_{j}(w)
$$

where $\omega_{j}$ are the harmonic measure functions defined in $\S 3, \mu_{j}(w)$ is a real valued function that is a linear combination of $S\left(a_{k}, w\right) S(w, a), k=1, \ldots, n-1$, and

$$
\lambda_{j}(a)=\int_{\zeta \in \gamma_{j}} \frac{|S(\zeta, a)|^{2}}{S(a, a)} d s
$$

is a function in $C^{\infty}(\bar{\Omega})$ that has the same boundary values as $\omega_{j}(a)$, i.e., equal to one on $\gamma_{j}$ and equal to zero on the other boundary components. The Ahlfors map is the principal ingredient of the main term, $|S(w, a)|^{2} / S(a, a)$ in the Poisson kernel; the other term, $\sum_{j=1}^{n-1}\left(\omega_{j}(a)-\lambda_{j}(a)\right) \mu_{j}(w)$, is in $C^{\infty}(\bar{\Omega} \times \bar{\Omega})$.

The Garabedian kernel can also be expressed in terms of the Ahlfors map. Let $z \in \Omega$ and $w \in b \Omega$ and use identity (2.1) and the fact that $\overline{f_{a}}=1 / f_{a}$ on $b \Omega$ to rewrite formula $(3.2)$ in the form

$$
L(z, w)=\frac{f_{a}(w)}{f_{a}(z)-f_{a}(w)}\left(c_{0} S(z, a) L(w, a)+\sum_{i, j=1}^{n-1} \bar{c}_{i j} S\left(z, a_{i}\right) L\left(w, a_{j}\right)\right) .
$$

Since both sides of this identity are holomorphic in $z$ and $w$, this identity holds for $z, w \in \Omega, z \neq w$. Note that the constants $c_{0}$ and $c_{i j}$ are the same as the constants in (3.2).

The complimentary kernel $\Lambda(z, w)$ to the Bergman kernel (see [1,page 134]), may also be expressed in terms of the Ahlfors map via

$$
\Lambda(w, z)=4 \pi L(w, z)^{2}+\sum_{i, j=1}^{n-1} \lambda_{i j} L\left(w, a_{i}\right) S(w, a) S\left(z, a_{j}\right) L(z, a),
$$


$z, w \in \Omega, z \neq w$.

Finally, we mention that the gradient of the Green's function on a finitely connected domain $\Omega$ with $C^{\infty}$ smooth boundary is composed of finitely many functions of one variable in $C^{\infty}(\bar{\Omega})$. It is shown in [2] that

$$
\frac{\partial G}{\partial \bar{w}}(z, w)=\pi\left(\frac{S(z, w) \overline{L(w, z)}}{S(z, z)}-i \sum_{j=1}^{n-1}\left(\omega_{j}(z)-\lambda_{j}(z) \overline{g_{j}(w)}\right)\right.
$$

for all $z, w \in \Omega, z \neq w$, where $g_{j}(w)$ is a linear combination of the holomorphic functions $S\left(w, a_{k}\right) L(w, a), k=1, \ldots, n-1$.

\section{REFERENCES}

1. S. Bell, The Cauchy transform, potential theory, and conformal mapping, CRC Press, Boca Raton, 1992.

2. Complexity of the classical kernel functions of potential theory, Indiana Univ. Math. J. 44 (1995), 1337-1369.

3. Math. J. 64 (1991), 1-26.

4. _ Recipes for classical kernel functions associated to a multiply connected domain in the plane, Complex Variables, Theory and Applications 29 (1996), 367-378.

5. M. Schiffer, Various types of orthogonalization, Duke Math. J. 17 (1950), 329-366.

Mathematics Department, Purdue University, West Lafayette, IN 47907 USA

E-mail address: bell@math.purdue.edu 